\title{
Prevalence and associated factors of anemia among children aged 6 to 59 months in Ethiopia: Evidence from the Ethiopian demographic and health survey
}

\author{
Ashenafi Abate Woya, Abay Kassa Tekile* \\ Department of Statistics, College of Science, Bahir Dar University, Bahir Dar, \\ Ethiopia
}

\begin{abstract}
Anemia is one of the most widely spread public health problems, especially in developing countries including Ethiopia. The aim of this study was to assess the prevalence and associated factors of anemia among children aged 6-59 months in Ethiopia. A community-based cross-sectional study (the Ethiopian Demography and Health Survey 2016) was used as a source of data. Participants were 8385 children aged from 6 to 59 months selected in a two-stage stratified cluster sampling. The level of hemoglobin was determined by HemoCue analyzer. The risk factors of anemia were computed by logistic regression $(\alpha=0.05)$. The result revealed that more than half $(57.3 \%)$ of children aged 6-59 months were found anemic of which $3.1 \%$ had severe anemia, $29.2 \%$ had moderate and $25 \%$ mild anemia. The anemia status of the children's mother (OR: 3.01, CI: 0.34, 6.75), living in Somali region (OR: 5.73, CI: 1.86, 17.71), living in rural areas (OR: 1.84, CI: $1.38,2.83$ ), age of study participants (OR: 0.82, CI: $0.29,1.45$ ) among 24-42 months old children (OR: 0.53, CI: 0.16,1.08) among 43-59 months old children, rich and medium parents (OR: 0.29, CI: 0.20,0.73), smoker mother (OR: 0.02, CI: $0.00,0.05)$ were the risk factors of anemia among Ethiopian children aged 6-59 months. The overall prevalence of anemia among Ethiopian children aged 6-59 months was high. It is argued that measures that prevent childhood illnesses and maternal anemia need to be put in place in order to reduce anemia among Ethiopian children.
\end{abstract}

Keywords: Anemia, Prevalence, Children, 6-59 months, Associated factors, Ethiopia

DOI: https://dx.doi.org/10.4314/ejst.v14i1.4

* Corresponding author: abaystat@gmail.com

(C) This is an Open Access article distributed under the terms of the Creative Commons Attribution License (http://creativecommons.org/licenses/CC BY4.0) 


\section{INTRODUCTION}

As one of the common blood disorders, anemia is a global public health problem among children. It affects 1.62 billion people globally (WHO, 2015). Approximately two-thirds of preschool children in Africa and South East Asia were anemic and more than half of the world's preschool-age children $(56.3 \%)$ reside in countries where anemia is a major public health problem (WHO, 2019). In 2011, WHO estimated 273.2 million children aged 6-59 months suffer from anemia globally. Sub-Saharan Africa is the most affected, with a prevalence of $62.3 \%$ (WHO, 2011). The World Health Organization (WHO) classified the burden of anemia as severe when prevalence is over $40 \%$, moderate when between 20 and $40 \%$, and mild when between 5 and 20\% (WHO, 2005). World Health Organization regional estimate shows that the highest proportion of anemic pre-school age children reside in Africa (Steel, 2017).

Anemia is also a major public health problem among preschool-aged children in Ethiopia. Childhood anemia has a substantial association with social, economic, psychological, and health-related problems. The evidence demonstrated that early childhood anemia is a strong predictor of adulthood anemia (Chen et al., 2013).

According to $2018 \mathrm{WHO}$ report, anemia is a serious global public health problem affecting mostly young children and pregnant women. In developing countries, the prevalence of anemia among preschool age children was $40 \%$ (WHO, 2015). The problem of anemia is alarmingly high in sub-Sahara Africa with a prevalence of 60.2 to $87.8 \%$ of children from 6 to 59 months (Magalhães and Clements, 2011).

According to Ethiopian Demographic and Health Survey (EDHS) reports, $54 \%$ of children were anemic in 2005 and $44 \%$ in 2011 (CSA, 2005, 2011). Of this, $21 \%$ of the children aged 6-59 months were mildly anemic, $20 \%$ were moderately anemic, and $3 \%$ were severely anemic.

Several factors affect the development of anemia among children aged 659 months. Demographic characteristics of mothers such as region and residence, wealth index, source of drinking water, hemoglobin level of the mother and the child's characteristics such as age, sex, and nutritional status are the major factors linked with anemia among children aged from 6-59 months (Kemal Nure et al., 2018).

Although a lot of research has been conducted on anemia among children in Ethiopia, there is still high prevalence of anemia among children in 
Ethiopia since strict preventive measures are not well addressed. Therefore, this study was aimed at determining the prevalence of anemia and identify risk factors associated with it among children aged 6-59 months in Ethiopia.

\section{MATERIALS AND METHODS}

\section{Study design, period and area}

A cross-sectional community-based survey was conducted from January 18, 2016 to June 27, 2016 from 2016 EDHS data. The data were collected by the Central Statistical Agency (CSA) at the request of the Federal Ministry of Health (FMOH). The 2016 EDHS used a two-staged stratified cluster sampling to ensure representativeness at national and regional levels. Initially, each region was stratified into urban and rural areas, yielding 21 sampling strata. Each stratum was subdivided into enumeration areas. Based on the 2007 Ethiopia population and housing census, and after stratification, a total of 645 enumeration areas (202 urban and 443 rural) were selected with probability proportional to enumeration area size. A household listing operation was carried out in all the selected enumeration areas from September to December 2015. A total of 28 households were selected from each cluster using the systematic random sampling technique from the household listing. A total of 8,385 children were recruited to this study.

\section{Hemoglobin determination}

Data on the health status of children were collected when the enumerators identified children in the selected households. Based on the consent of parents or caretakers to the test, level of blood hemoglobin (Hgb) was measured from children aged from 6 to 59 months. Capillary blood samples were collected using a finger prick or a heel prick in the case of children aged 6-11 months. The level of Hgb was determined on site using a portable HemoCue analyzer and the result was reported in $\mathrm{g} / \mathrm{dl}$. Accordingly, the result was interpreted as anemic $(\mathrm{Hgb}<11.0 \mathrm{~g} / \mathrm{dl})$, mild $(\mathrm{Hgb}=10.0-10.9 \mathrm{~g} / \mathrm{dl})$, moderate $(\mathrm{Hgb}=7.0-9.9 \mathrm{~g} / \mathrm{dl})$, and severe $(\mathrm{Hgb}<7.0$ $\mathrm{g} / \mathrm{dl}$ ) using the WHO criteria. 
Study variables

Dependent variable: Anemia status among children aged 6-59 months. Independent variables: Mother's anemia status, region, residence, wealth index, mother's smoking status, religion, age and sex of the children and educational status of the mother.

\section{Data processing and statistical analysis}

This study employed ordinal logistic regression (OLR) model to identify risk factors of anemia among children aged 6-59 months. As the dependent variable was ordinal (anemia status of children), an OLR modelproportional odds model (POM) was developed to find predictors of anemia at $p$-value $=<0.05$. The data were analyzed using STATA version 14 software.

\section{Odds ratio}

The odds ratio is a value which measures the strength of the effect of each independent variable in the model on the log odds of the dependent variable (anemia status of children). The odds of anemia in children is defined as the ratio of the number of anemic children to the number of none anemic children. That is the odd of the anemic is given by:

$$
\begin{gathered}
\text { Odds(children anemic status }=\frac{\operatorname{Pr}(\text { anemic children })}{\operatorname{Pr}(\text { nonanemic })} \\
=\frac{\operatorname{Pr}(\text { anemic children })}{1-\operatorname{Pr}(\text { anemic children })}
\end{gathered}
$$

The odds of the response are multiplied by $e^{\beta}$ for every unit increment of $\mathrm{x}$. That is the odds at level $\mathrm{x}+1$ equals the odds at $\mathrm{x}$ multiplied by $e^{\beta}$, where an odds less than one indicates that the occurrence is less likely than nonoccurrence.

\section{Assumptions of ordinal logistic regression}

In this study, a proportional odds model (POM) was employed, by using the logits of anemia severity (i.e., logit of severe, logit of mild, logit of moderate and logit of none) are represented as a parallel linear function of the predictor (residual). This indicates for each cumulative logit, the (residual) parameter of the models is the same except for the intercept. Consequently, the odds ratio of the categories (severe, mild, moderate and none anemia) is homogenous since the odds ratio has a value. Therefore, it can be concluded that there is considerable evidence that leads to accepting 
the assumption of proportional odds model. The proportional odds model is used in the analysis of ordinal data, the coefficients of the explanatory variables in the model is interpreted as the logarithm of the ratio of the odds of the response variable. This means that estimates of this odds ratio, and corresponding confidence intervals, can be easily found from the fitted model. The interpretations of risk factors were considered significant at $5 \%$ level.

\section{Ethical consideration}

The survey protocol for the 2016 EDHS was reviewed and approved by the Federal Democratic Republic of Ethiopia Ministry of Science and Technology and the Institutional Review Board of Inner-City Fund (ICF) International.

\section{Operational definitions}

The following WHO operational definitions were used:

Stunted: It refers to low height-for-age, when a child is short for his/her age but not necessarily thin.

Under-weight: Under-weight refers to low weight-for-age, when a child can be either thin or short for his/her age.

Wasted: Wasted refers to low weight-for-height where a child is thin for his/her height but not necessarily short.

Wealth Index: The Wealth Index is a composite measure of the cumulative living standard of a household. It is calculated using data a household owns (a selected set of assets), such as television, bicycles, and cars; dwelling characteristics such as flooring material; type of drinking water source; and toilet and sanitation facilities.

Region: Ethiopia is a federation subdivided into ethno-linguistically based Regional States and chartered cities. There are nine regional states and two chartered cities in Ethiopia. 


\section{RESULTS}

\section{Demographic characteristics of children and their mothers}

A total of 8385 children aged 6-59 months were included in this study. The median age of children was 32 months. The majority of the children $(n=4362,52.0 \%)$ were from mothers aged 25-34 years. Most of these children were from rural areas $(\mathrm{n}=7525,89.7 \%)$ and from Oromia region $(n=3654,43.6 \%)($ Table 1$)$.

\section{Prevalence of anemia among children}

The prevalence of anemia among children aged 6-59 months was 4808 (57.3\%). Of which, 2099 (25.0\%) had mild anemia, $2448(29.2 \%)$ moderate and $261(3.1 \%)$ severe anemia. Rural children $(29.8 \%)$ and children whose families belong to poor wealth index $(32.3 \%)$ were moderately anemic. Likewise, children whose mothers aged from 15-34 years $(32.9 \%)$ had moderate anemia while $2.8 \%$ were with severely anemic (Table 1). About $39.8 \%$ of mothers whose children were aged 6-59 months suffered from moderate and $7.2 \%$ severe anemia. Three-fourth of children aged from 6-23 months were anemic. Of these, $40.6 \%$ had moderate and $3.8 \%$ severe anemia. Some $57.5 \%$ of the children were wasted, $57.6 \%$ of them stunted and $58 \%$ of underweight were anemic (Table 1). The prevalence of anemia was higher among children in Somali $(83.1 \%)$ and Afar regions (74.5\%) (Figure 1).

\section{Multivariable analysis}

On multivariable analysis, anemia among children aged from 6-59 months was significantly associated with children from mildly anemic mothers (AOR: 1.98, CI: 1.21, 3.26), moderately anemic mothers (AOR: 3.01, CI: $1.34,6.75)$, children living in Somali region $(\mathrm{AOR}=5.75, \mathrm{CI}: 1.86,17.71)$, Harari $((\mathrm{AOR}=3.88, \mathrm{CI}: 1.31,11.51)$ and Dire Dawa $(\mathrm{AOR}=6.85, \mathrm{CI}$ : $1.88,24.90)$, living in rural area $(\mathrm{AOR}=1.84,95 \% \mathrm{CI}: 1.38,1.95)$, age of study participants (OR:0.82, CI:0.29,1.45) among 24-42 months old children (OR:0.53,95\% CI:0.16,1.08) among 43-59 months old children, rich and medium parents (OR:0.29, CI:0.20,0.73), smoker mother (OR:0.02, CI:0.00, 0.05).Children from mildly anemic mothers were 1.98 times more likely to be anemic than children from mothers who were not anemic. On the other hand, children from moderately anemic mothers were about 3 times more likely to have anemia than children from mothers who had normal Hgb level (Table 2). 
Table 1. Socio-demographic characteristics of children and prevalence of anemia among children aged 6-59 months in Ethiopia (EDHS, 2016)

\begin{tabular}{|c|c|c|c|c|c|c|}
\hline Variables & Categories & $\begin{array}{c}\text { Frequency } \\
\text { N (\%) }\end{array}$ & $\begin{array}{c}\text { Mild } \\
\text { anemia } \\
\text { N (\%) } \\
\end{array}$ & $\begin{array}{c}\text { Moderate } \\
\text { anemia } \\
\text { N (\%) } \\
\end{array}$ & $\begin{array}{l}\text { Severe } \\
\text { anemia } \\
\text { N (\%) }\end{array}$ & $\begin{array}{l}\text { Overall } \\
\text { anemia } \\
\text { N }(\%)\end{array}$ \\
\hline \multirow[t]{3}{*}{ Child age (months) } & $6-23$ & $2878(34.3)$ & $795(27.5)$ & $1168(40.6)$ & $108(3.8)$ & $2069(71.9)$ \\
\hline & $24-42$ & $2949(35.2)$ & $710(24.1)$ & $834(28.3)$ & $116(3.9)$ & $1660(56.3)$ \\
\hline & $43-59$ & $2558(30.5)$ & $595(23.4)$ & $445(17.4)$ & $37(1.4)$ & $1079(42.2)$ \\
\hline \multirow[t]{2}{*}{ Sex of children } & Male & $4339(51.7)$ & $1059(24.4)$ & $1283(29.6)$ & $143(3.3)$ & $2486(57.3)$ \\
\hline & Female & $4046(48.3)$ & $1040(25.7)$ & $1165(28.8)$ & $117(2.9)$ & $2322(57.4)$ \\
\hline \multirow[t]{11}{*}{ Region } & Addis Ababa & 161(1.9) & $32(19.7)$ & $43(27.0)$ & $3(1.9)$ & $78(48.7)$ \\
\hline & Afar & $82(1.0)$ & $23(27.6)$ & $35(42.9)$ & $3(4.0)$ & $61(74.5)$ \\
\hline & Amhara & $1647(19.6)$ & $380(23.1)$ & $287(17.4)$ & $33(2.0)$ & $701(42.6)$ \\
\hline & Benishangul & $89(1.1)$ & $21(24.1)$ & $16(18.5)$ & $1(0.8)$ & $38(43.4)$ \\
\hline & Dire Dawa & $31(0.4)$ & $7(23.0)$ & $12(39.7)$ & $3(9.3)$ & $22(71.9)$ \\
\hline & Gambela & $20(0.2)$ & $5(24.2)$ & $6(32.0)$ & $0(0.8)$ & $11(56.9)$ \\
\hline & Harari & $15(0.2)$ & $4(24.1)$ & $6(37.4)$ & $1(5.6)$ & $10(67.1)$ \\
\hline & Oromia & $3654(43.6)$ & $973(26.6)$ & $1275(34.9)$ & 141(3.9) & $2388(65.4)$ \\
\hline & SNNPR & $1774(21.2)$ & $447(25.2)$ & $433(24.4)$ & $25(1.4)$ & $904(51.0)$ \\
\hline & Somali & $343(4.1)$ & $59(17.3)$ & $184(53.7$ & $42(12.1)$ & $285(83.1)$ \\
\hline & Tigray & $570(6.8)$ & $149(26.1)$ & $150(26.3)$ & $9(1.6)$ & $308(54.0)$ \\
\hline \multirow[t]{2}{*}{ Residence } & Urban & $860(10.3)$ & $207(24.1)$ & $209(24.30$ & $10(1.2)$ & $427(49.6)$ \\
\hline & Rural & $7525(89.7)$ & $1892(25.1)$ & $2239(29.8)$ & $250(3.3)$ & $4381(58.2)$ \\
\hline \multirow[t]{5}{*}{ Religion } & Orthodox & $2898(34.6)$ & 683(23.6) & $635(21.9)$ & $57(2.0)$ & $1375(47.5)$ \\
\hline & Catholic & $79(0.9)$ & $31(39.1)$ & $13(16.6)$ & $0(0.0)$ & $44(55.7)$ \\
\hline & Protestant & $1864(22.2)$ & $492(26.4)$ & $485(26.0)$ & $24(1.3)$ & $1001(53.7)$ \\
\hline & Muslim & $3365(40.1)$ & $861(25.6)$ & $1241(36.9)$ & $158(4.7)$ & $2260(67.2)$ \\
\hline & Other & $104(1.2)$ & $11(10.2)$ & $50(47.9)$ & $20(19.2)$ & $80(77.3)$ \\
\hline
\end{tabular}




\begin{tabular}{|c|c|c|c|c|c|c|}
\hline \multirow[t]{2}{*}{ Wealth index } & Poor & $3916(46.7)$ & $995(25.4)$ & $1267(32.3)$ & $193(4.9)$ & $2454(62.7)$ \\
\hline & Middle and rich & $4469(53.3)$ & $1104(24.7)$ & $1181(26.4)$ & $68(1.5)$ & $2354(52.7)$ \\
\hline \multirow{2}{*}{$\begin{array}{l}\text { Mother's smoking } \\
\text { status }\end{array}$} & No & $8315(99.2)$ & $2087(25.1)$ & $2428(29.2)$ & $257(3.1)$ & $4771(57.4)$ \\
\hline & Yes & $70(0.8)$ & $12(17.3)$ & $20(28.9)$ & $4(5.8)$ & $36(52.1)$ \\
\hline \multirow{4}{*}{$\begin{array}{l}\text { Mother's anemia } \\
\text { status }\end{array}$} & Not anemic & $5861(78.0)$ & $1440(24.6)$ & $1511(25.8$ & $120(2.0)$ & $3070(52.4)$ \\
\hline & Mild & $1875(16.6)$ & $503(26.8)$ & $698(37.2$ & $82(4.3)$ & $1283(68.4)$ \\
\hline & Moderate & $521(4.5)$ & $115(22.0)$ & $207(39.8)$ & $38(7.2)$ & $360(69.0)$ \\
\hline & Severe & 127(0.9) & $42(32.6)$ & $31(24.6$ & $22(17.1)$ & $95(74.2)$ \\
\hline \multirow[t]{2}{*}{ Wasting } & wasted & $5461(65.1)$ & $1386(25.4)$ & $1554(28.5$ & $198(3.6)$ & $3139(57.5)$ \\
\hline & Not wasted & $2924(34.9)$ & $713(24.4)$ & $894(30.6$ & $62(2.1)$ & 1669(57.) \\
\hline \multirow[t]{2}{*}{ Stunting } & stunted & $6995(83.4)$ & $1766(25.3)$ & $2047(29.3$ & $213(3.0)$ & $4027(57.6)$ \\
\hline & Not stunted & $1390(16.6)$ & $333(23.9)$ & $401(28.8$ & $48(3.4)$ & $781(56.2)$ \\
\hline \multirow[t]{2}{*}{ Underweight } & underweight & $7188(85.7$ & $1810(25.2)$ & $2118(29.5)$ & $237(3.3)$ & $4166(58.0)$ \\
\hline & Not underweight & $1197(14.3)$ & $290(24.2)$ & $329(27.5)$ & $23(2.0)$ & $642(53.7)$ \\
\hline \multirow{3}{*}{$\begin{array}{l}\text { Mother's education } \\
\text { status }\end{array}$} & No education & $5421(64.6)$ & $1240(22.9)$ & $1566(28.9)$ & $160(3.0)$ & $2965(54.7)$ \\
\hline & Primary & $2103(25.1)$ & $594(28.3)$ & $578(27.5)$ & $74(3.5)$ & $1246(59.3)$ \\
\hline & $\begin{array}{l}\text { Secondary and } \\
\text { above }\end{array}$ & $861(10.3)$ & $265(30.8)$ & $304(35.3)$ & $27(3.1)$ & $596(69.2)$ \\
\hline \multirow[t]{3}{*}{ Mother's age } & $15-24$ & $2100(25.0)$ & $488(23.3)$ & $691(32.9)$ & $60(2.8)$ & $1239(59.0)$ \\
\hline & $25-34$ & $4362(52.0)$ & $1079(24.7)$ & $1168(26.8)$ & $139(3.2)$ & $2387(54.7)$ \\
\hline & $35-49$ & $1923(22.9)$ & $532(27.7)$ & $589(30.6)$ & $62(3.2)$ & $1182(61.5)$ \\
\hline Total & & 8385 & $1659(19.8)$ & $2448(29.2)$ & $261(3.1)$ & $4808(57.3)$ \\
\hline
\end{tabular}




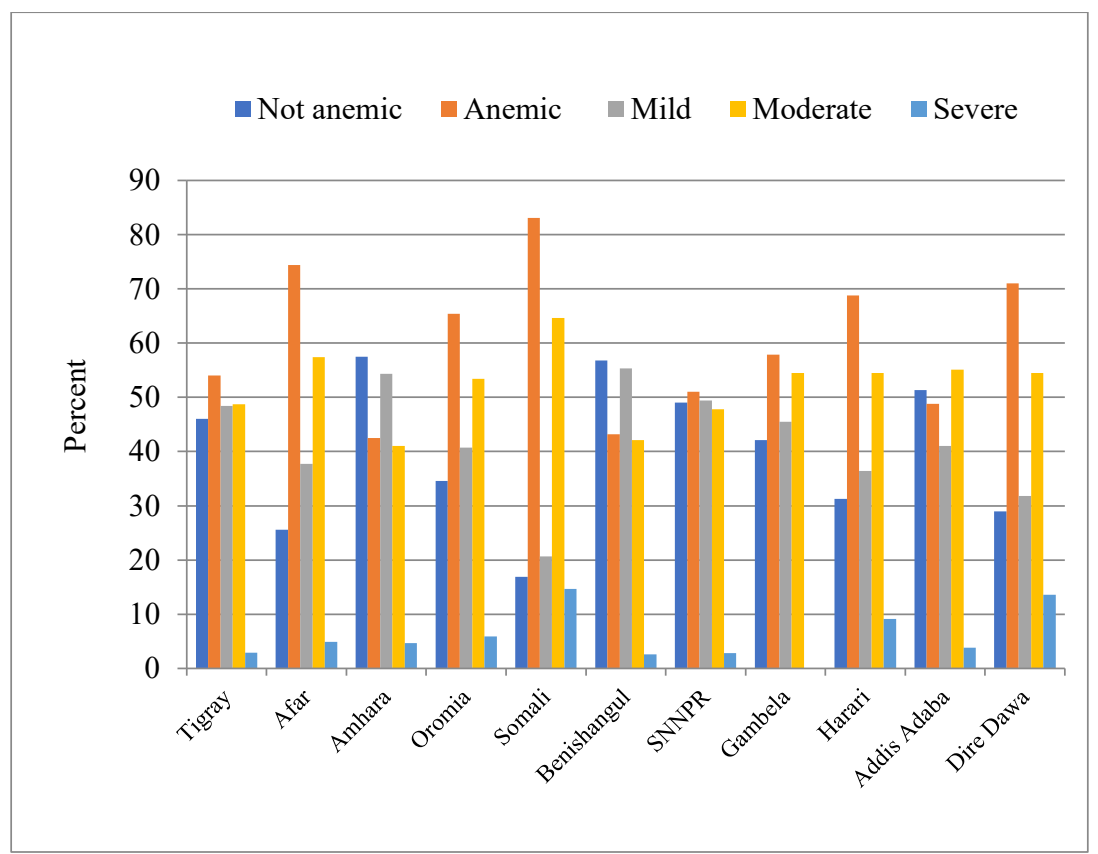

Figure 1. Regional distribution of anemia among children aged 6-59 months in Ethiopia

The odds of developing anemia among children from Somali region was 5.75 times that of Tigray. The odds of developing anemia among children from Harari region was 3.88 times that of Tigray region. The odds of developing anemia among children from Dire Dawa federal city administration was 6.85 times more likely than that of Tigray region. The odds of developing anemia among children who resided in rural areas were 1.84 times higher than those living in urban area.

The odds of developing anemia among children aged 24-42 months and 43-59 months were only 0.82 and 0.53 times that of children aged from 523 months, respectively. Odds of being anemic of children of medium and rich wealth index households was only 0.29 times that of children from poor households. 
Table 2. Multivariable analysis on the factors Associated with Anemia among Children at the age of 6-59 months in Ethiopia (EDHS, 2016)

\begin{tabular}{|c|c|c|c|c|}
\hline Variables & Category & COR & AOR [95\% CI] & $p$-value \\
\hline \multirow{4}{*}{$\begin{array}{l}\text { Anemia } \\
\text { status of } \\
\text { mothers }\end{array}$} & Not anemic (ref) & & & \\
\hline & Mild & 1.92 & $1.98[1.21,3.26]$ & 0.007 \\
\hline & Moderate & 2.80 & $3.01[1.34,6.75]$ & 0.008 \\
\hline & Severe & 5.14 & $3.67[0.50,26.68]$ & 0.200 \\
\hline \multirow[t]{11}{*}{ Region } & Tigray (ref) & & & \\
\hline & Afar & 2.43 & $2.16[0.76,6.16]$ & 0.149 \\
\hline & Amhara & 0.65 & $0.75[0.39,1.44]$ & 0.393 \\
\hline & Oromia & 1.57 & $1.67[0.72,3.88]$ & 0.235 \\
\hline & Somali & 4.72 & $5.73[1.86,17.71]$ & 0.002 \\
\hline & Benishangul & 0.65 & $0.55[0.22,1.39]$ & 0.209 \\
\hline & SNNPR & 0.90 & $1.88[0.85,4.19]$ & 0.121 \\
\hline & Gambela & 1.30 & $0.67[0.26,1.72]$ & 0.400 \\
\hline & Harari & 1.88 & $3.88[1.31,11.51]$ & 0.015 \\
\hline & Addis Adaba & 0.88 & $1.01[0.31,3.29]$ & 0.984 \\
\hline & Dire Dawa & 2.66 & $6.85[1.88,24.90]$ & 0.003 \\
\hline \multirow[t]{2}{*}{ Residence } & Urban (ref) & & & \\
\hline & Rural & 1.37 & $1.84[1.38,1.83]$ & 0.041 \\
\hline \multirow{3}{*}{$\begin{array}{l}\text { Child age } \\
\text { (months) }\end{array}$} & $6-23$ (ref) & & & 0.000 \\
\hline & $24-42$ & 1.04 & $0.82(0.29,0.45)$ & 0.001 \\
\hline & $43-59$ & 0.76 & $0.53(0.16,0.78)$ & 0.014 \\
\hline \multirow[t]{2}{*}{ Wealth index } & Poor (ref) & & & \\
\hline & Middle and rich & 0.54 & $0.29[0.20,0.73]$ & 0.003 \\
\hline \multirow{8}{*}{$\begin{array}{l}\text { Smoking } \\
\text { status } \\
\text { Religion }\end{array}$} & No (ref) & & & \\
\hline & Yes & 0.88 & $0.02[0.00,0.05]$ & 0.001 \\
\hline & Orthodox (ref) & & & \\
\hline & Catholic & 1.19 & $1.40[0.46,4.28]$ & 0.551 \\
\hline & Protestant & 1.32 & $0.81[0.36,1.85]$ & 0.620 \\
\hline & Muslin & 2.74 & $1.24[0.57,2.67]$ & 0.583 \\
\hline & Traditional & 1.72 & $0.98[0.09,10.33]$ & 0.978 \\
\hline & Other & 2.08 & $16.34[1.88,141.54]$ & 0.011 \\
\hline
\end{tabular}

Ref $=$ Reference category, $\mathrm{COR}=$ Crude Odds Ratio, $\mathrm{AOR}=$ Adjusted Odds Ratio, $\mathrm{CI}=$ Confidence interval

\section{DISCUSSION}

The prevalence of anemia in the current study was $57.3 \%$ which was higher than a previous report of the national prevalence estimate of $2005(54 \%)$ and 2011 (44\%) EDHS reports (CSA, 2005, 2011). The percentage of 
anemia among children decreased with time (from 2005 to 2011). The proportion of anemia among children, however, increased from 2011 to 2016.

The analysis of the present study revealed that about one-third of children aged 6-59 months were moderately anemic. This was consistent with the 2005 EDHS report. However, compared to the 2011 EDHS report, it was higher. The level of severe anemia among children in the present study was the same as the one in 2005 and 2011 EDHS reports (CSA, 2011).

This study showed that the presence of anemia among mothers was significantly associated with anemia among children at the age of 6-59 months. This could be linked to their lower consumption of home iron due to poverty (Semedo et al., 2014). Households need better access to food and higher cash income to allow them better diet and better access to medical care (Pollitt, 2001; Semedo et al., 2014). Compared to children from medium and rich households, children from households with poor wealth index had higher odds of being anemic. Percentage of children with anemia is significantly higher among low-income families (Kawo et al., 2018). Many previous studies indicated that poverty is a significant factor of anemia (Baxter et al., 2008). Poverty directly related to the anemic status of children and the economic status of the family determined the children's anemic status. Thus, to control and manage children's anemic status, improving the economy of the Ethiopian rural households is crucial. The proportion of anemia among children aged 6 to 59 months was significantly higher among those residing in rural than urban areas $(p<0.041)$. This finding is consistent with studies reported previously in Ethiopia and Brazil (Kindie Fentahun, 2016; Osório et al., 2001). The low consumption of iron-containing foods among children in rural areas due to poverty, poor sanitation and water supplies might be the reasons for the high prevalence of anemia among rural children.

This study also showed that anemia significantly varied from region to region among children. Children living in Somali region, Harari and Dire Dawa were more likely to develop anemia than those from Tigray region. This is comparable with previous studies that was carried out in Ethiopia and in Brazil (Kindie Fentahun, 2016; Osório et al., 2001). The reasons for this might be the variation of environmental, socio-economic, and cultural practices among regions. 
Compared with children aged 6-23 months, the odds of developing anemia among children aged 24-42 months and 43-59 months was lower. Similar findings were reported before in Ethiopia (Kemal Nure et al., 2018). The main reason might be that older children receive a diet that is rich and sufficient in iron as a result of more varied diet, which could prevent the occurrence of anemia.

The odds of being severely, moderately or mildly anemic was higher among children whose mothers smoked, a finding that is consistent with previous study in Ethiopia (Barakat et al., 2014).

\section{Limitations of the study}

Because of the cross-sectional nature of the EDHS data, the cause-effect and temporal relationship could not be established. Likewise, important factors such as dietary intake and behavioral factors were not available in the EDHS and therefore it was not possible to incorporate these variables in the analysis. Moreover, EDHS was a questionnaire-based survey and relied on the memory of the respondents, and as such, recall bias in the results might be a weakness for this study.

\section{CONCLUSION}

In Ethiopia, the prevalence of anemia among children at the age of 6-59 months was high. Children from anemic mothers, regional variation (Somali, Harari and Dire Dawa), rural residence, child's age, and the wealth status of mothers were predictor variables for anemia among children. Therefore, the Ministry of Health together with concerned collaborators should put more effort to decrease the prevalence of anemia among children through maternal education and provide supportive treatments to both pregnant mothers and children.

\section{ACKNOWLEDGMENTS}

First of all, the authors would like to express their sincere gratitude to study participants for their willingness to participate in the study. We acknowledge the Ethiopia Central Statistical Agency for giving us permission to use the data for our study. The manuscript was edited by 
Berhanu Engidaw (Assistant professor), English Department, Bahir Dar University.

\section{Consent for publication}

Not applicable.

\section{Competing interests}

We the authors declare that we have no competing interest.

\section{REFERENCES}

Barakat, A.A., Nada, K.H and Ezzat, D.A. (2014). Prevalence and determining factors of anemia and malnutrition among Egyptian children. Indian Journal of Medical Sciences 67: 168-177.

Baxter, R., Hastings, N., Law, A and Glass, E. (2008). The economic impact of anemia in Peru. Lima: Group for the analysis of development and action against hunger. Animal Genetics 39(1): 561-563.

Chen, M., Su, T., Chen , Y., Hsu, J., Huang, K and Chang, W. (2013). Association between psychiatric disorders and iron deficiency anemia among children and adolescents: A nationwide population-based study. BMC Psychiatry 13(161): 1-8.

CSA (2005). Central statistical agency and orc macro, Ethiopia demographic and health survey, Central Statistical Agency, Addis Ababa, Ethiopia.

CSA (2011). Central statistical agency and orc macro, Ethiopia demographic and health survey, Central Statistical Agency, Addis Ababa, Ethiopia.

Kemal Nure, Zeytu Gashaw and Negusse Yohannis (2018). Multilevel analysis of determinants of anemia prevalence among children aged 6-59 months in Ethiopia: classical and Bayesian approaches. Anemia 2018: 1-13.

Kindie Fentahun (2016). Determinants of severity levels of anemia among children aged 659 months in Ethiopia: Further analysis of the 2011 Ethiopian demographic and health survey. BMC Nutrition 2(1): 1-8.

Magalhães, R.J.S and Clements, A.C. (2011). Spatial heterogeneity of haemoglobin concentration in preschool-age children in sub-Saharan Africa. doi: 10.2471/BLT.10.083568.

Osório, M.M., Lira, P.I., Batista-Filho, M and Ashworth, A. (2001). Prevalence of anemia in children 6-59 months old in the state of Pernambuco, Brazil. Revista Panamericana de Salud Pública 10: 101-107.

Pollitt, E. (2001). The developmental and probabilistic nature of the functional consequences of iron-deficiency anemia in children. The Journal of Nutrition 131(2): 669S-675S.

Semedo, R.M., Santos, M.M., Baião, M.R., Luiz, R.R and da Veiga, G.V. (2014). Prevalence of anaemia and associated factors among children below five years of age in Cape Verde, West Africa. Journal of Health, Population, and Nutrition 32(4): 646.

Steel, N. (2017). Global, regional, and national age-sex specific mortality for 264 causes of death, 1980-2016: A systematic analysis for the global burden of disease study 2016. Lancet 390(10100): 1151-1210. 
WHO (2005). Worldwide prevalence of anaemia 1993-2005:WHO global database on anemia.

WHO (2011). Haemoglobin concentrations for the diagnosis of anaemia and assessment of severity. Vitamin and mineral nutrition information system. Geneva, World Health Organization

WHO (2015). Global anaemia prevalence and number of individuals affected. Reference Source.

WHO (2019). The Global prevalence of anemia. World Health Organization, Geneva, Switzerland. 